\title{
DESIGN OF VIBRATION-DAMPERS FOR STEEL CHIMNEYS WITH LATEST FEATURES
}

\author{
Manoj Kr Gupta .', Bajpai V.K. ${ }^{2}$, Garg T.K. ${ }^{3}$ \\ ${ }^{1}$ Research Scholar, Department of Mechanical Engineering, National Institute of Technology, Kurukshetra, India \\ 2,3 Department of Mechanical Engineering, National Institute of Technology, Kurukshetra, India \\ Email: ${ }^{1}$ manoj•gupta280@yahoo.com
}

\begin{abstract}
In the past there have been attempts to control wind response of chimneys using strakes, perforated screens, rubber mountings, hanging chain dampers and tuned mass dampers. However, a systematic and detailed study on latest designed vibrationdampers at chimney top is not seen in literature. In the present paper, principle of the damping system is discussed. Mass and stiffness of the damper are calculated. Amplitude of the top of chimney during vortex shedding is calculated. Results thus obtained have been discussed. Some latest damping systems have also been presented for authenticity.
\end{abstract}

Keywords: Vortex shedding, Degree of freedom, Stiffness, Resonance frequency, Critical damping, Scruton Number, Strouhal Number

\section{INTRODUCTION}

Economy in chimney design can be achieved by controlling its wind response. A chimney should have an adequate factor of safety against collapse, either along wind, in response to fastest gust it is likely to experience, or across wind, in response to vortex shedding. In addition, violent movements of chimney are to be avoided, even if consequent chimney stresses are acceptable. Such large deflections not only pose a threat to the safety and integrity of the structure, but also affect sensitive equipment and human occupants. To alleviate this problem, within economic constraints, concept of structural control has become inevitable, which can be implemented if considered early enough in the design process. Structural control may be implemented through a set of vibration dampers $[1,2]$. These dampers generate an opposing force that counters the motion of the basic structure to which it is attached. Different types of damping available are viscous, dry friction or coulomb, solid or structural and slip or interfacial damping [3]. In this paper, some of the efforts towards controlling the vibrations in chimneys using

various vibration dampers are presented. These dampers are suitable for self supported and guy wired supported tall steel chimneys.

\section{PRINCIPLE OF DAMPING SYSTEM}

The movements of the chimneys in cross wind direction are caused by vortices. The forces due to these vortices can have the same frequency as the chimney and resonance can easily occur. The amplitude and stresses of the chimney can become large and can cause fatigue of the steel $[4,5]$. A damping structure can reduce the movements considerably, because of counter forces with the same frequency. The theory of damping system is illustrated in figure1.

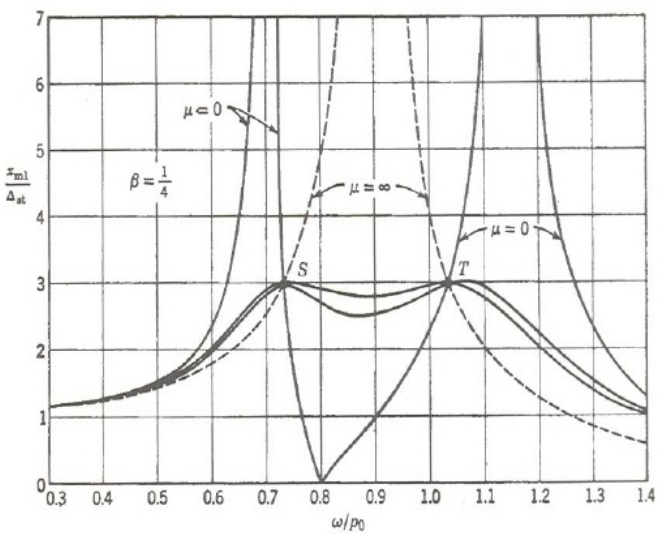

Fig. 1. Dynamic response of system with and without a damper [3]

The vertical axis shows the ratio between the dynamic $X^{\mathrm{ml}}$ and the static $\Delta^{\text {st }}$ movement of the chimney. On the horizontal axis is the frequency of loading divided by the resonance frequency of the chimney. The dotted curve in figure 1 is the dynamic behavior of the chimney without a damper. The amplitude becomes very large if the frequency of the load $(\omega)$ becomes equal to the frequency of the chimney $\left(\rho^{0}\right)$. If the damping structure has been an optimal designed, then the amplitude is restricted to the curves with a horizontal tangent at the points $S$ and $T$. The amplitude will not go above three times static amplitude [3]. This can be considered as an excellent reduction of movements and stresses or an increase of the damping. The conditions for changing the single degree of freedom system of the chimney without a damping structure into the system with maximum amplitudes of $S$ and $T$ are discussed below. The ratio of resonance frequencies $f_{\text {chimney }}$ of the main system and the damping system $\mathrm{f}_{\text {damper }}$ is as: 


$$
\begin{gathered}
\frac{f_{\text {damper }}}{f_{\text {chimney }}}=\frac{1}{\sqrt{1+\frac{m_{2}}{m_{1}}}} \\
\mathrm{~m}_{1}=\frac{\int_{0}^{h} m(z) Y^{2}(z) d z}{Y_{\max }^{2}}
\end{gathered}
$$

The stiffness of the damping mass is:

$$
\mathrm{k}_{2}=\mathrm{k}_{1} \frac{1}{1+\frac{m_{1}}{m_{2}}}
$$

The damping:

$$
\begin{aligned}
& c_{2}=\frac{c_{2 c r}}{1+\frac{m_{2}}{m_{1}}} \sqrt{\frac{3 m_{2}}{8 m_{1}}} \\
& c_{2 \mathrm{cr}}=2 \sqrt{m_{2} k_{2}}
\end{aligned}
$$

$\mathrm{m}_{2}$ and $\mathrm{k}_{2}$ are mass and stiffness of the damper.

This damping ratio of a dynamic system with one degree of freedom is explained below:

>If the ratio is 1 , then the movement of the mass after being displaced will go back to the rest situation, without passing this position.

$>$ If the ratio is 0 , then there is no damping and the mass will go over the rest situation to the same distance on the other side. The movement will continue.

$>$ If the movement is only $50 \%$ of the starting value after $n$ cycles, then the damping ratio is $0.11 / \mathrm{n}$. This is a simple way to determine the damping ratio of a single system of freedom: move it out of the rest situation and count the number of movements until the movement is reduced by $50 \%$. The damping ratio is then $0.11 / \mathrm{n}$.

The critical wind velocity is:

$\mathrm{V}_{\text {crit }}=c_{\mathrm{r}} \mathrm{fd}(6)$

Where $c^{r}=5$ ( $c^{r}$ is the inverse of the Strouhal Number)

\section{MOVEMENT OF CHIMNEY}

The amplitude of the top of the chimney during vortex shedding can be calculated. Introducing the
Strouhal Number $[6,8]$ as 0.2 , the maximum amplitude $y$ and the chimney as a single degree of freedom system with stiffness $\mathrm{k}$, mass $\mathrm{mh} / 4$ and damping $\mathrm{c} / \mathrm{c}_{\mathrm{cr}}$ loaded by a force $F$ in resonance with the chimney, then the movement of the top is:

$y=F /\left(2 k c / c^{c r}\right)$

The frequency is:

$$
f=\frac{1}{2 \pi} \sqrt{\frac{k}{m h / 4}}
$$

And the amplitude is:

$$
\begin{gathered}
y / d=\frac{8 C_{L}}{S_{c r}} \\
S_{c r}=\frac{4 \pi m \frac{c}{c_{c r}}}{\rho d^{2}}
\end{gathered}
$$

Where, $m$ is the mass per unit length of the top third. The velocity of the top of the chimney is given by:

$v=\omega y=8.1 \pi C_{L} d f / S_{c r}$

The critical wind velocity is given by $5 \mathrm{fd}$. If the Scruton number is larger than 7 the movement of the chimney has an influence on the loading. The load is increased. For Scruton numbers larger than 15 , the movements become smaller than $5 \%$ of the diameter and the influence of the movements can be neglected [10]. Normally the Scruton number is above 15 if the damping ratio $c / c^{c_{1}} 1 / 23 \%$.

\section{FEATURES OF DAMPERS}

Damper is fitted to chimney at the topmost portion. Attachment of damper with chimney is shown in Figure 2.

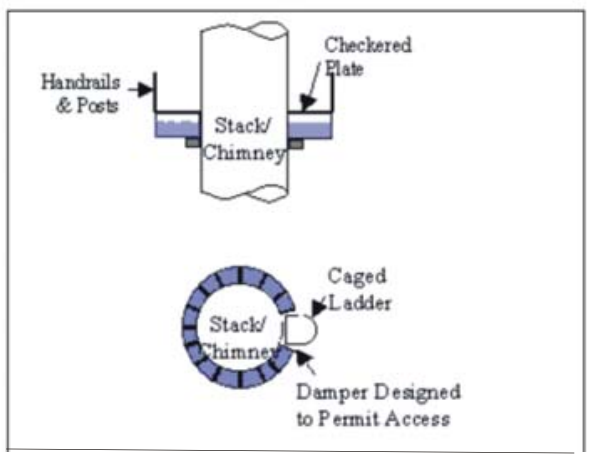

Fig.2. Arrangement of damper with stack 


\section{A. Dimensions of Dampers}

- The damper must create a force to compensate for the force of the vortex.

The force is given by

$1 / 2 \rho{ }^{\circ 2} C^{L} d h={ }^{*} 1.25^{*} 25^{*} d{ }^{3} h \omega^{2} / 4 \pi^{2}=0.08 \omega^{2} d^{3} h$

- The force of the damper $=$ me $\omega^{2}$

- The force of the vortex is compensated if : $m e=0.08 d^{3} h$

- Where $m$ is the mass of the damper and $e$ is the eccentricity out of rest. If the movement of the mass is supposed to be close to $0.08 \mathrm{~m}$, then the mass in $\mathrm{kg}$ must be about: $m \geqslant d^{3} h$

\section{EXAMPLES OF DAMPING SYSTEMS}

The following damping systems are latestly designed [9]. Each system has its advantages and disadvantages.

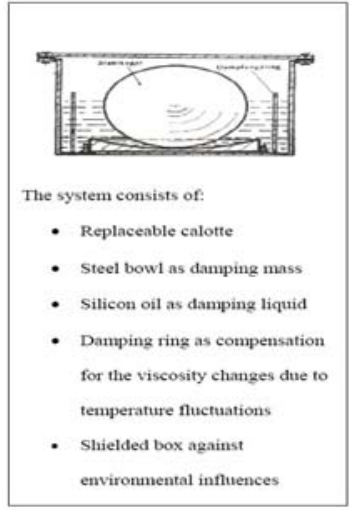

Fig. 3. Traetner System

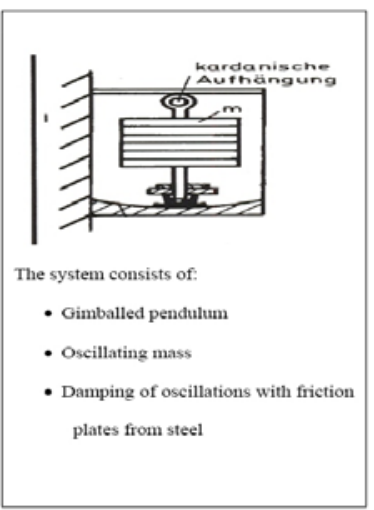

Fig. 5. Petersen System

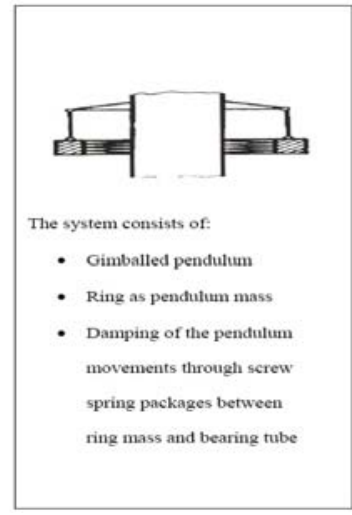

Fig. 4. Hirsch System

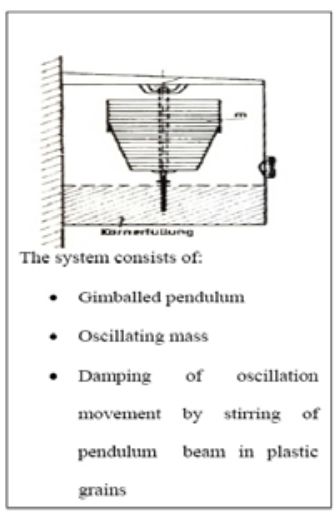

Fig.6. Langer System

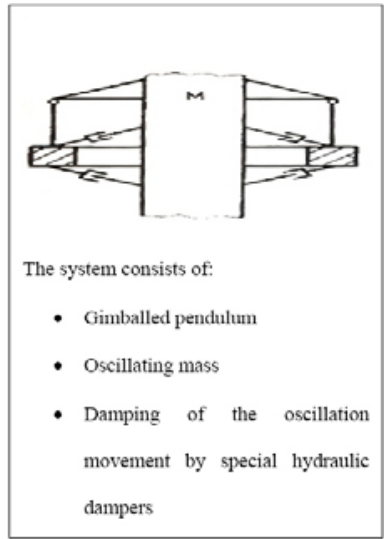

Fig. 7. Reutlinger System

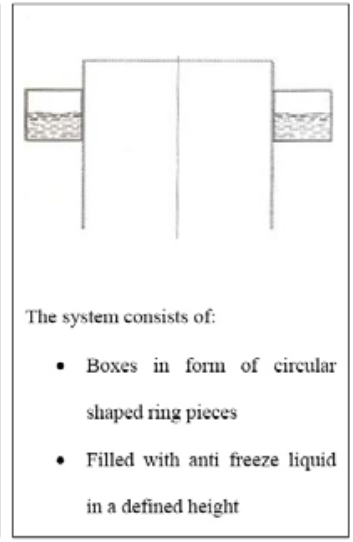

Fig. 8. Verwiebe System

\section{INACCURATE TUNING OF A DAMPING STRUCTURE}

The tuning of a damper at the optimal frequency and damping is not always possible and the frequency of a chimney and its damping can change. Calculations have been made to find the effect of this accuracy. If the resonance frequency of the damper is $10 \%$ outside of the optimal value the damping of the chimney is reduced by $20 \%$. This is not important, because the minimum required damping is $3 \%$ of the critical damping. The damping of the damping system must be close to 0.1 to 0.2 . If the damping is more than this value the damping of the chimney is hardly changed $[9,11,12]$.

\section{CONCLUSION}

The minimum required damping to prevent cross wind vibrations is $3 \%$ of the critical damping [10]. Dampers made with water need an anti freezing protection. Tall chimneys can be damped by using the non linear coils or hydraulic shock absorbers. Regular tests by measuring the variations of the stresses at the base of the chimney are advised. The tests make it possible to determine the damping and the effect of the damping system.

\section{REFERENCES}

[1] B. N. Pritchard, 1984, "Steel chimney oscillations: a comparative study of their reported performance versus predictions using existing design techniques", Engineering Structures, vol. 6, pp.315323.

[2] O. R. Jaiswal, Vasala Srinivas, 2006, "Pendulum type tuned mass damper to control along and across-wind response of tall chimneys", Third National Conference on Wind Engineering, N. Delhi, pp.162-171. 
[3] Den Hartog, 1985, "Mechanical Vibrations", Dover Publications: Newyork.

[4] B.J.Vickery, Dec1983," Simplified approaches to the evaluation of the across-wind response of chimneys", Journal of Wind Engineering and Industrial Aerodynamics, Vol 14, Issue 1-3, pp.153166.

[5] M. Zdravkovich, 1981, "Review and classification of various aerodynamic and hydrodynamic means for suppressing vortex shedding", Journal of Wind Engineering and Industrial Aerodynamics, Vol 7 , Issue 2, pp.145-189.

[6] IS: 6533-1989 (Part II), "Code of Practice for Design and Construction of Steel Chimney, Structural Aspect", Bureau of Indian Standards.

[7] www.mecaenterprises.com

[8] Francesco Ricciardelli, 2001, "On the amount of tuned mass to be added for reduction of the shedding-induced response of chimneys", Journal of Wind Engineering and Industrial Aerodynamics, vol. 89 (14-15), pp.1539-1551.

[9] Klaus K. Kaemmer, 2005, The CICIND Chimney Book, International Committee on Industrial Chimneys: Germany.
[10] H. Van Koten, Calculation of Damping of Chimneys, CICIND Report 17-1.

[11] Hans Ruscheweyh, Galemann Thomas, 1996, "Full-scale measurements of wind-induced oscillations of chimneys", Journal of Wind Engineering and Industrial Aerodynamics, Vol 65, pp.55-62.

[12] R. Ciesielski, A. Flaga, J., Kawecki 1996, "Aerodynamic effects on a non-typical steel chimney $120 \mathrm{~m}$ high", Journal of Wind Engineering and Industrial Aerodynamics, Vol 65, pp.77-86.

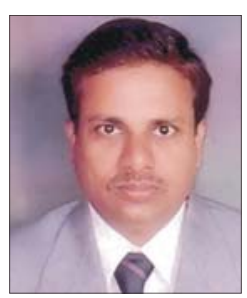

Manoj Kr Gupta is a Research Scholar at the Deparment. of Mechanical Engineering, NIT Kurukshetra, India. He obtained his Mechanical Engineering degree from REC Kurukshetra and a postgraduate in design from NIT Kurukshetra. He has over eight years of industrial experience. He is an expert in Steel Chimneys, Vibration Engineering and Machine Design. He has more than 10 research papers in International \& National Journals and Conferences to his credit. 